\title{
Post-processing of "Pulse code modulation" encoder output to downtick bit rates for voice transmission
}

\author{
M.Sravan Reddy ${ }^{1}$, Dr.D.Vishnuvardhan ${ }^{2}$ \\ Student, ECE Department, JNTUA College of Engineering, Pulivendula, India ${ }^{1}$ \\ Assistant professor, ECE Department, JNTUA college of Engineering, Pulivendula, India ${ }^{2}$
}

\begin{abstract}
This paper presents a technique for Post-processing of the digital output obtained by encoding an analog voice signal using an 8-bit Pulse code modulation (PCM) encoder sampled at $8 \mathrm{KHz}$, to decrease the bit rate from 64 Kbps to $56 \mathrm{Kbps}$ without decreasing the number of encoding levels or sampling frequency. Further, the paper the adavantages of such reduction in practical applications is discussed in detail. It is observed that using the proposed technique there is a reduction in bit rate by $12 \%$ approximately and the number of voice calls a channel of given bandwidth can support is increased by around $15 \%$.
\end{abstract}

Keywords: Voice, Coding, Channel bandwidth, bit rates.

\section{INTRODUCTION}

Pulse CODE Modulation is the technique widely used for transmission of human voice over the telecommunication Networks. There are two PCM codecs, namely PCM $\mu$-law which is standardized for use in North America and Japan, and PCM A-law for use in Europe and the rest of the world. ITU-T G.711 was standardized by ITU-T for PCM codecs in 1988 [1].In PCM the input analog voice signal is sampled at 8000 samples per second, because the range of frequencies in voice is $0 \mathrm{~Hz}$ to $4 \mathrm{KHz}$; by nyquist theorem sampling at $8 \mathrm{KHz}$ is sufficient to reproduce the analog speech signal at receiver without aliasing. For detailed description of PCM Refer [2]. In PCM 7-bit encoding (128 quantization levels) is used for encoding each voice sample and an extra bit (1 or 0 ) as Most significant bit (MSB) used to indicate sign. Hence, a total of 8-bits are used to encode each speech sample. Since, the analog voice signal is sampled at 8000 samples per second and each sample is encoded using 8 bits, therefore, $8000 \times 8=64000$ bits are transferred per second. Thus, the bit rate of PCM is $64 \mathrm{Kbps}$, which is the standard PCM bit rate widely used in telecommunications. To reduce bit rate of PCM, either sampling frequency is to be reduced or number of bits used for encoding (encoding levels) must be reduced. There is a trade-off when we try to reduce the bit rate of PCM. If sampling frequency is reduced then bit rate decreases but accuracy will decrease [2]. Similarly, decrease in number of coding levels will reduce bit rate but increases quantization error. Decreasing bit rates will increase the number of voice calls a channel of given bandwidth can support, but with decrease in accuracy or increase in quantization error.

In this paper, we propose a post-processing technique that aims at reducing the transmission bit rate of PCM by 8 kbps without any reduction in sampling frequency or number of coding levels. The Section II will describe the

method used in this paper, Section III describes the advantages of the proposed method and finally, Section IV concludes this paper.

\section{PROPOSED METHOD}

In original PCM, each voice sample is encoded using 8bits and hence 8-bits are transmitted through the channel for each sample. In the proposed method a "logical bit reduction" (LBR) block as shown in Fig. 1 is added to PCM encoder that performs post-processing of PCM output to reduce the number of bits to be transmitted for each sample from 8-bits to 7-bits.

Analog

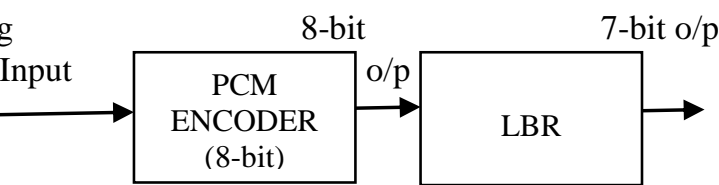

Fig. 1. PCM encoder with LBR

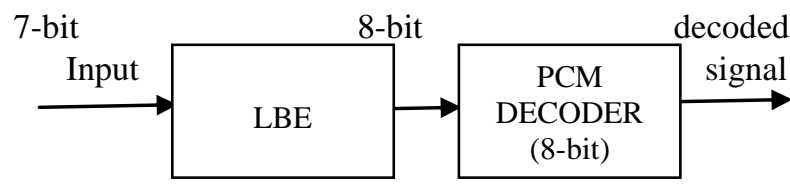

Fig. 2. PCM decoder with LBE

Thus, the bit rate will be reduced to $8000 \times 7=56000$ samples per second. Though, 7-bits are used for transmission, but, at the receiver the original 8-bits are recovered using "logical bit expansion" (LBE) block added before PCM decoder as shown in Fig. 2.

\section{A. Categorizing 4-bit binary combinations}

The "logical bit reduction" block takes into account that, 4-bit binary combinations exhibit certain patterns which can be used to segregate them into various categories. For 4-bits, $2^{4}=16$ binary combinations [3] are possible. Each 
combination can be classified into one of the 3 categories defined as:

Category - I: A 4-bit binary combination falls into this category,

If $[($ bit $-1=$ bit -4$)$ and $($ bit $-2=$ bit -3$)]$

Example: 1001, 1111, 0110.

Category - II: A 4-bit binary combination falls into this category,

If $[($ bit $-1 \sim=$ bit -4$)$ and (bit $-2 \sim=$ bit -

Example: 1010, 0101, 1100.

Category - III: A 4-bit binary combination falls into this category,

If $[($ bit $-1=$ bit -4$)$ and (bit $-2 \sim=$ bit -3$)]$

or

If [(bit-1 = bit -4$)$ and (bit $-2=$ bit -3$)]$

Example: 1011,0111,0100,1101.

Table -1 shows all the 16 possible binary combinations and their corresponding category. Table -1 is the base for bit reduction using LBR block.

TABLE - 1: CATEGORIZING 4-BIT BINARY COMBINATIONS

\begin{tabular}{|c|c|}
\hline Combination & Category \\
\hline 0000 & I \\
\hline 0001 & III \\
\hline 0010 & III \\
\hline 0011 & II \\
\hline 0100 & III \\
\hline 0101 & II \\
\hline 0110 & I \\
\hline 0111 & III \\
\hline 1000 & III \\
\hline 1001 & I \\
\hline 1010 & II \\
\hline 1011 & III \\
\hline 1100 & II \\
\hline 1101 & III \\
\hline 1110 & III \\
\hline 1111 & I \\
\hline
\end{tabular}

\section{B. Bit reduction using LBR block}

For each voice sample PCM gives an 8-bit digital output as shown in Fig. 3.

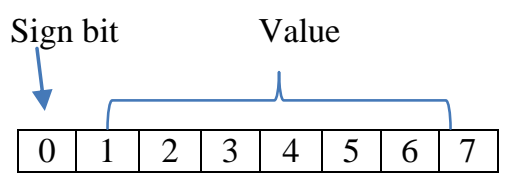

Fig. 3. 8-bit PCM output format for each sample.

As seen from Fig. 3. each sample is represented using 8 bits. The $0^{\text {th }}$ bit, also called Most significant bit (MSB) indicates sign (" 1 " for positive value and " 0 " for negative value) and remaining 7 bits indicate numerical value of the sample. These 8-bits of each sample are given as input to the "logical bit reduction" block, which then gives a 7-bit output to be transmitted for each sample. Let $\mathrm{X}$ be the 8 bit binary code for a particular sample given as input to the
LBR block and $X^{\prime}$ be the output of LBR. The algorithm for bit reduction using LBR:

1. Divide $X$ into two halves, say $Y$ and $Z$ each of 4-bits. $Y$ contains first 4 MSB bits which include the sign bit and $\mathrm{Z}$ contains the remaining bits.

2. Let Y' and Z', each of 3-bit be the code obtained after reducing $\mathrm{Y}$ and $\mathrm{Z}$.

3. Determine the categories of $\mathrm{Y}$ and $\mathrm{Z}$ using Table -1 .

4. If ( $\mathrm{Y}$ belongs to category I) then $\mathrm{Y}^{\prime}=\left(1\left[1^{\text {st }}\right.\right.$ bit of $\left.\mathrm{Y}\right]\left[2^{\text {nd }}\right.$ bit of $\left.\left.\mathrm{Y}\right]\right)$. Else if ( $\mathrm{Y}$ belong to category II) then $\mathrm{Y}^{\prime}=\left(0\left[1^{\text {st }}\right.\right.$ bit of $\left.\mathrm{Y}\right]\left[2^{\text {nd }}\right.$ bit of $\left.\left.\mathrm{Y}\right]\right)$.

Else ( $\mathrm{Y}$ belong to category III) then

(i) Invert the LSB (right most bit) of Y.

(ii) Go to step -3 to determine category of $Y$, as (i)

Converts Y from category III to either category I or II 5. Similarly, Perform step -3 to determine $Z$ '.

$6 . X^{\prime}=\left[Y^{\prime} Z^{\prime}\right]$, which is a six bit value.

7. Let $\mathrm{K}$ be the $7^{\text {th }}$ bit (LSB) of $\mathrm{X}^{\prime}$. In step - 3, if Y belongs to category - III, then $\mathrm{K}=1$ is appended as $7^{\text {th }}$ bit (right most bit) to the 6-bit value obtained in step - 5. Else, $\mathrm{K}=0$ is appended as $7^{\text {th }}$ bit.

8. Final coded value $X^{\prime}=\left[Y^{\prime} Z^{\prime} K\right]$, which is a 7-bit value.

In this way, an 8-bit code is reduced to 7-bit code for each sample and is transmitted through the channel. In step - 3, if Y belongs to category - III, then, LSB of Y is inverted. This means if LSB is ' 0 ' it is changed to ' 1 ' and viceversa. This logically means that the coding level of the sample is either increased or decreased by 16 coding levels. Suppose, if an encoded sample belongs to coding level " 128 " then, due to bit inversion its level changes to " 112 " which is an error. Hence, ' $\mathrm{K}$ ' is used to indicate the LBE block whether coding levels are changed or not. $K=1$ means LSB of $\mathrm{Y}$ at encoder is inverted and must be inverted at the decoder also to get back the original bit. This ensures that coding levels of the samples are not changed. The algorithm is illustrated with an example. Assume, $X=10110101$ be the encoded output of a particular sample using 8-bit PCM. Dividing $\mathrm{X}$ into two halves, $\mathrm{Y}=1011$ and $\mathrm{Z}=0101$. $\mathrm{Y}$ belongs to category III and hence we invert the last bit, which gives $Y=1010$. Now, Y changes to category - II. Therefore, the reduced value of $Y$ will be $Y^{\prime}=010$. Similarly for $Z$ which belongs to category - II, the reduced value is $Z Z^{\prime}=001$. Since, $Y$ belongs to category - III, the $7^{\text {th }}$ bit is $K=1$. The final reduced 7-bit code to be transmitted in this case will be $\mathrm{X}^{\prime}=\left[\mathrm{Y}^{\prime} \mathrm{Z}^{\prime} \mathrm{K}\right]=0100011$

\section{Bit expansion using LBE block}

At the receiver, the 7-bit code " $\mathrm{X}$ " for each sample is given as input to the LBE block to reconstruct back the original 8-bit PCM encoded value. Actual Y and Z each of 4-bit are reconstructed from $X$ '. Let " $R$ " be 8-bit output reconstructed using LBE block. The algorithm used in LBE block for bit expansion is as follows:

1. Divide $X^{\prime}$ into 3 parts, $Y^{\prime}$ (first three most significant bits), Z' (next three most significant bits) and $\mathrm{K}$ (the last bit or Least significant bit). 
2. If $\left(\operatorname{MSB}\left(Y^{\prime}\right)=1\right)$ then

Y belongs to category - I

$Y(4-b i t)=\left[\left(2^{\text {nd }}\right.\right.$ bit of $\left.Y^{\prime}\right)\left(3^{\text {rd }}\right.$ bit of $\left.Y^{\prime}\right)\left(3^{\text {rd }}\right.$ bit of $\left.Y^{\prime}\right)$ $\left(2^{\text {nd }}\right.$ bit of $\left.\left.Y^{\prime}\right)\right]$

$$
\begin{aligned}
& \text { Else if }\left(\mathrm{MSB}\left(\mathrm{Y}^{\prime}\right)=0\right) \text { then } \\
& \text { Y belongs to category }- \text { II } \\
& \mathrm{Y}(4-\mathrm{bit})=\left[\left(2^{\text {nd }} \text { bit of } \mathrm{A}\right)\left(3^{\text {rd }} \text { bit of } \mathrm{A}\right) \sim\left(3^{\text {rd }} \text { bit of } \mathrm{A}\right)\right. \\
& \left.\sim\left(2^{\text {nd }} \text { bit of } \mathrm{A}\right)\right] \\
& \text { 3. Repeat Step } \left.-2 \text { for } \mathrm{X}^{\prime} \text { to get back } \mathrm{X} \text { (4-bits }\right) \text {. } \\
& \text { 4. If }(\mathrm{K}=1) \text { invert LSB of } \mathrm{Y} \text {. Else no inversion. } \\
& \text { 5. Reconstructed code, } \mathrm{R}=[\mathrm{Y} \mathrm{Z}] \text {. }
\end{aligned}
$$

Consider $X^{\prime}=0100011$ obtained for $X$ in the previous example.

Divide $\mathrm{X}^{\prime}$ into $\mathrm{Y}^{\prime}, \mathrm{Z}^{\prime}$ and $\mathrm{K}$ respectively. So, $\mathrm{Y}^{\prime}=010, \mathrm{Z}$ ' $=001$ and $\mathrm{K}=1$. Since MSB of $\mathrm{Y}$ ' is " 0 ", $\mathrm{Y}$ belongs to category - II. Therefore, $\mathrm{Y}=1010$. But $\mathrm{K}=1$, so the LSB of $Y$ must be inverted, which gives, $Y=1011$. Similarly, MSB of $Z$ ' is also ' 0 ". So, $Z$ belongs to category - II and hence $\mathrm{Z}=0101$. The reconstructed 8-bit PCM encoded value is $\mathrm{R}$ $=[\mathrm{Y} \mathrm{Z}]=10110101$. " $\mathrm{R}$ " which is the output of LBE block is equal to " $\mathrm{X}$ " which is the input to LBR block. The original 8-bit PCM code for the sample is reconstructed from the 7-bit reduced code. The output of the LBE is given as input to PCM decoder which performs the usual decoding to reconstruct the voice signal. The LBR block reduces the number of bits to be transmitted through the channel from 8-bits to 7-bits without reducing the sampling frequency or the number of encoding levels. Hence, the bit rate is reduced from $64 \mathrm{Kbps}$ to $54 \mathrm{Kbps}$. The codec is still an 8-bit PCM with $8 \mathrm{KHz}$ sampling frequency.

\section{ADVANTAGE}

Addition of LBR block to PCM offers certain advantages in practical applications like, efficient utilization of channel bandwidth and decrease in bit rates.

\section{A. Channel Bandwidth Utilization}

With the addition of LBR block to PCM, the bit rate is reduced by $8 \mathrm{Kbps}$. Thus, for a channel of give bandwidth we can allocate more number of voice calls. Let us consider a channel of bandwidth $1 \mathrm{MB}$ between two points $\mathrm{A}$ and $\mathrm{B}$. The number of voice calls that can be supported with PCM having a bit rate of $64 \mathrm{Kbps}$ are $1 \mathrm{MB} / 64 \mathrm{Kbps}$ $=15$ voice calls. Now, with PCM having bit rate of 56 $\mathrm{Kbps}, 1 \mathrm{MB} / 56 \mathrm{kbps}=18$ voice calls can be supported.

The increase in number of voice calls will be approximately $15 \%$. We could have achieved this without adding LBR block to PCM. But, that requires either lowering of sampling frequency or encoding levels with a trade - off between accuracy, quantization error and bandwidth. But, by adding LBR block there is no need to reduce sampling frequency or encoding levels and the codec is still an 8-bit PCM with $8 \mathrm{KHz}$ sampling frequency and accuracy is maintained. Fig. 4. Shows the comparison between PCM and PCM with LBR in terms of number of voice calls supported for a channel of given bandwidth.

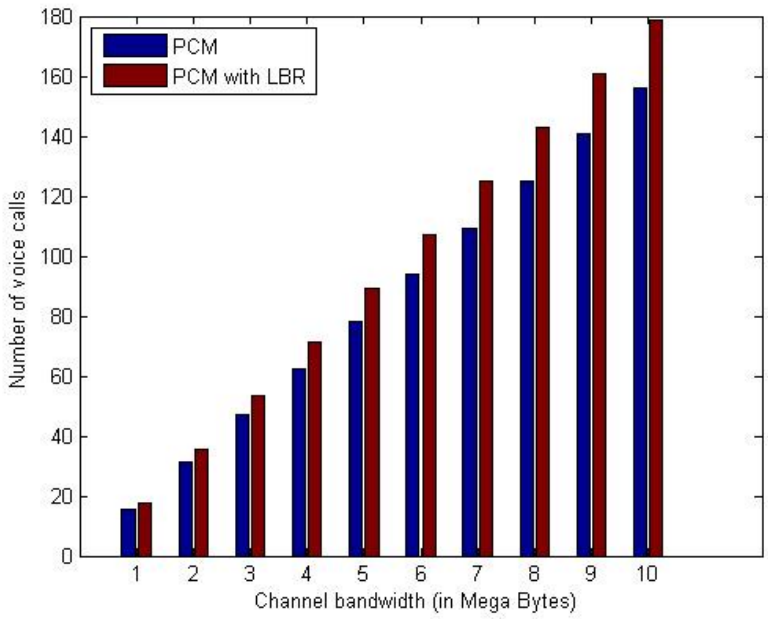

Fig. 4. Comparing PCM and PCM with LBR in terms of Number of voice calls supported with a channel of given bandwidth

T-1 carrier [4] is the most widely used digital transmission service in the US, Canada and Japan. In these countries, it consists of 24 separate channels using PCM signals (8-bit) with TDM at an overall bit rate of $1.544 \mathrm{Mbps}$. They originally use copper wires but now also include optical and wireless media. It is much more reliable than an analog modem. Depending on what they are doing, a T1 line can generally handle quite a few people. With the proposed method, using same $\mathrm{T}-1$ carrier transmission setup but PCM signals (7-bit) the overall bit rate reduces to $1.344 \mathrm{Mbps}$. The reduction is approximately $12 \%$. Hence, more users can be supported with the same 24 channel setup.

\section{CONCLUSION}

In this paper, two new blocks LBR and LBE are added to the existing 8-bit PCM codec for post-processing of PCM digital output to reduce the bit rate. With the addition of these blocks, the transmission bit rate is reduced by 8 Kbps without any reduction in sampling frequency or number of encoding levels. The number of voice calls that can be supported by a channel of give bandwidth is increased by $15 \%$ approximately and bit rate is decreased by $12 \%$ approximately.

\section{ACKNOWLEDGMENT}

The authors would like to thank the members and the faculty of JNTUA college of Engineering, Pulivendula for their support towards this research.

\section{REFERENCES}

[1] Goodall, W.M., "Telephony by pulse code modulation," in Bell System Technical Journal, The, vol.26, no.3, pp.395-409, July 1947

[2] L. Sun et al., Guide to Voice and Video over IP, Computer Communications and Networks, Springer-Verlag London 2013.

[3] Morris Mano, M. and Michael Ciletti, D, Digital Design. Fourth edition. 2008

[4] T1-Telecommunications, ANSI Standard T1.102-1993. 